\title{
MODELING OF OCCURRENCE OF SURFACE DEFECTS OF C45 STEEL WITH GENETIC PROGRAMMING
}

\author{
MODELIRANJE POJAVA POVRŠINSKIH NAPAK PRI JEKLU C45 Z \\ GENETSKIM PROGRAMIRANJEM
}

\author{
Miha Kovačič ${ }^{1}$, Robert Jager ${ }^{2}$ \\ 1Štore Steel, d. o. o., Železarska cesta 3, 3220 Štore, Slovenia \\ ${ }^{2}$ Laboratory for Multiphase Processes, University of Nova Gorica, Vipavska 13, 5000 Nova Gorica, Slovenia \\ miha.kovacic@store-steel.si \\ Prejem rokopisa - received: 2013-12-09; sprejem za objavo - accepted for publication: 2015-02-18
}

doi:10.17222/mit.2013.304

\begin{abstract}
Carbon steel C45 with an increased content of carbon is used for tempering in the automotive industry for highly stressed parts (axles, shafts), machine parts, screws, drills for wood, axes, knives, hammers and similar. In the present work an attempt of analyzing the influences of different steelmaking parameters is presented. On the basis of the monitored data about the casting-temperature changes, the total oxygen, the number of added aluminum rods, the chemical analyses before and after steelmaking, the added lime, the aluminum-cored wire, the calcium-silicon-cored wire, the sulphur-cored wire, the rolling dimensions, the casting speed, the opening of the ladle nozzle with oxygen and surface defects (scrap fraction) on rolled bars, a mathematical model was obtained with the help of the genetic programming method. The results show that the most influential parameters for the surface-defect occurrence on the C45 steel are the opening of the ladle nozzle with oxygen and aluminum. On the basis of the results, the steelmaking technology was changed. Instead of the aluminium-killed steelmaking technology the aluminium-calcium-free (ACF) steelmaking technology was used. The batches from an aluminium-calcium-free steelmaking period statistically have a significantly lower level of surface defects (scrap fraction). The scrap fraction was reduced from the average of $68.45 \%$ to $1.92 \%$ - by more than 35 times.

Keywords: mechanical engineering, metallurgy, steel, C45 steel, making steel, casting steel, steel plant, billet surface defects, genetic programming
\end{abstract}

Jeklo C45 je ogljikovo jeklo za poboljšanje s povečano koncentracijo ogljika. Uporablja se za obremenjene dele v avtomobilski industriji (osi, gredi), za dele strojev, vijake, svedre za les, sekire, nože, kladiva in podobno. V članku je predstavljen poskus analize vplivov različnih parametrov pri postopku izdelave omenjenega jekla. Na podlagi zbranih podatkov o spremembi livne temperature, aktivnega kisika (kisik v talini), dodanih aluminijevih palic, analiz kemijskih elementov med izdelavo jekla in po njej, dodanega apna, dodanega aluminija, kalcij-silicija ter žvepla v obliki žice, dimenzij valjanca, hitrosti litja, podžiganja in podatkov o površinskih napakah (delež izmeta) valjanih palic smo izdelali matematični model z genetskim programiranjem. Rezultati modeliranja genetskega programiranja so pokazali, da sta podžiganje in aluminij tista parametra, ki najbolj vplivata na nastajanje napak v jeklu C45 in imata torej največji vpliv na izmet. Na podlagi rezultatov se je spremenila tehnologija izdelave jekla. Namesto tehnologije z dodatkom aluminija se je uporabila tehnologija aluminium-calcium-free (ACF). Šarže, izdelane v obdobju izdelave jekla $s$ to tehnologijo, imajo statistično značilno manj površinskih napak (izmeta). Izmet se je $v$ povprečju zmanjšal iz $68,45 \%$ na $1,92 \%$ - več kot 35 -krat.

Ključne besede: strojništvo, metalurgija, jeklo, jeklo C45, izdelava jekla, ulivanje jekla, jeklarna, površinske napake na gredici, genetsko programiranje

\section{INTRODUCTION}

The basic concerns about the development of the continuous-casting technology are associated with finding the source generating surface defects and taking proper measures to prevent and remedy them where appropriate. ${ }^{1-3}$

A literature review shows that there are three basic ways of modeling surface defects:

- experimental approach ${ }^{4}$,

- computational fluid dynamics ${ }^{1-3}$ and

- artificial-intelligence approach which effectively

combines the above-mentioned approaches. ${ }^{5-10}$

The authors ${ }^{5,6}$ propose an interaction between the numerical heat-transfer model and the artificial-intelligence heuristic-search method, linked to a knowledge base for the continuous casting. A two-dimensional heat-transfer model was developed using the finitedifference method and applied to real continuous-casting conditions. The heuristic search method, aided by a knowledge base, explores the technological and metallurgical parameter settings in order to find optimized cooling conditions, resulting in a defect-free billet production with the minimum metallurgical period.

The paper by Tirian et al. ${ }^{7}$ describes a neuralnetwork-based strategy for crack prediction aimed at improving the steel-casting process performance by decreasing the number of crack-generated failure cases. A neural system for estimating the crack-detection probability was designed, implemented, tested and integrated into an adaptive control system.

A simulated annealing-optimization algorithm was used for a multicriteria optimization procedure to determine appropriate process-parameter values for producing 
quality products in a continuous-casting system. ${ }^{8}$ A total of 17 critical-quality conditions were identified; these had to be satisfied to prevent defect formations during the casting. An objective function, formulated as a loss function, was used so that all 17 critical conditions were satisfied simultaneously.

The article by Sanz-García et al. ${ }^{9}$ deals with three successful experiences gained from genetic algorithms and the finite-element method in order to solve engineering-optimization problems in connection with the surface-defect occurrence during continuous casting.

A set of metamodels was developed to satisfy the necessary process conditions connected to one or more continuous-casting parameters. ${ }^{10}$ The values of these parameters were determined so that all the process conditions were satisfied simultaneously, ensuring that the product had the desired quality.

This paper discusses the use of the genetic-programming method for predicting the occurrence of surface defects on rolled C45 steel, used for highly stressed parts in the automotive industry, with respect to the influences of several steelmaking parameters. Genetic programming is one of the more general and recently developed approaches of evolutionary algorithms. Similarly to some other machine-learning approaches such as artificial neural networks, genetic algorithms, particle-swarm optimization and gravitational search algorithm (see examples ${ }^{11-14}$ ), genetic programming can be used for solving a wide spectrum of engineering and other problems.

The problem is described in Section 2. In the subsequent section the effect of the proposed concept and the results of the defect-occurrence prediction are presented. A practical implementation of the modeling is presented in Section 4 and, finally, the main contributions of the performed research and guidelines for further research are given in the last section.

\section{EXPERIMENTAL BACKGROUND}

Steelmaking begins with scrap melting in an electric-arc furnace. After the melting of the scrap and carburizing agents, the carbon carriers, in general, are coke, anthracite, graphite and slag additives, which regulate the basicity, viscosity, thermal and electric conductivities, desulphurization, dephosphorization, neutrality towards the furnace fireproof linings and capability to filter non-metallic inclusions. ${ }^{1,2}$

The melting bath heated up to the tapping temperature according to further treatment procedures is discharged into the casting ladle after the electric-arc-furnace melting.

The standard steelmaking procedure (in a ladle furnace) for C45 consists of:

1. Melt-temperature measurement. Depending on the temperature the melt is additionally heated.
2. Visual checking of the slag's viscosity and amount. The slag should be properly dense. In the case of not having enough slag, lime and bauxite should be added. The argon flux should also be appropriate.

3. Chemical analysis.

4. On the basis of the chemical composition, corrective alloying is performed and argon stirring is also consequently increased.

5. If necessary, aluminum and sulfur wires are added.

6. Chemical analysis.

7. On the basis of the chemical composition, fine corrective alloying is performed (CaSi and aluminum wires). During the alloying moderate argon stirring is performed. The melt should also be properly covered with slag due to a possible melt reoxidation. In the end a sulfur wire is added.

8. Melt-temperature measurement.

After achieving the proper melt temperature in the melting bath the billets are continuously cast. The melting bath flows through the sliding-gate system and ladle shroud towards the tundish.

During the casting it may be necessary to use the oxygen lance to cut through the ladle slide gate and pour the liquid steel through it. In good steelmaking practice, we want to have a minimum number of such batches because the oxygen that is blown into the melt causes a re-oxidation of the melt and impurities may be formed. ${ }^{15}$

After filling up the tundish, the mould filling system with tundish stoppers and submerged pouring tubes is established. Billets with a square section of $180 \mathrm{~mm}$ are cast. After reaching a certain melting-bath level the potentiometer starts the flattening system which drags a billet out of the mould. In this way continuous casting is established. The billet goes through the cooling zone toward the gas cutters where it is cut and laid off onto the cooling bed.

The cooled-down billets are reheated, according to the prescribed temperature, in the continuous-heating furnace. After the heating the billets are hot rolled in a strand of rolls. Steel bars are cooled on the cooling bed. After the cooling they are cut with hot shears to different lengths.

In line with the customer specifications, after the rolling the cut bars are inspected on the automatic control line (Figure 1) equipped with three inspection units:

- anti-mixing control (FÖRSTER MAGNATEST, eddy current),

- surface-defect control (FÖRSTER CYRCOFLUX, magnetic-flux leakage) and

- internal-defect control (KRAUTKRAMER ROWA 90/160, ultrasound).

The minimum detectable surface-defect depth and length are $0.15 \mathrm{~mm}$ and $12.5 \mathrm{~mm}$, respectively. Regarding the inspection speed and type of the ultrasonic head, the minimum detectable inner-defect sizes are $0.8 \mathrm{~mm}$ and $1.2 \mathrm{~mm}$. Due to blind areas and the type of the 


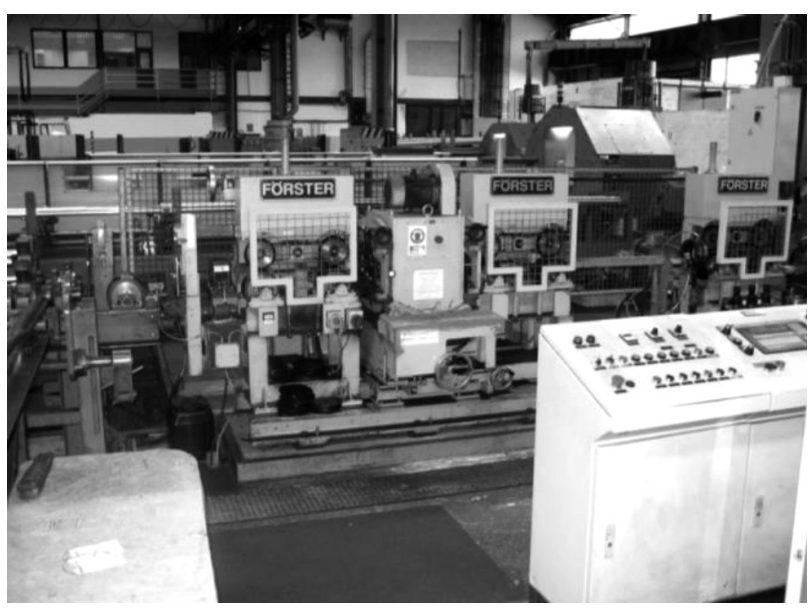

Figure 1: Automatic control line in Štore Steel Ltd.

Slika 1: Samodejna kontrolna linija v Štore Steel, d. o. o.

internal defects, the control unit detects $85 \%$ of the material volume.

The data for the analysis was collected on the basis of 34 batches of the C45 steel consecutively inspected (automatic control line) in Store Steel Ltd. (Table 1) from January to December 2010. The data was taken from the technological documentation of the cast batches and from the chemical archive. The goal was to get as wide a range of influential parameters as possible. These are:

- Active oxygen $\mathrm{O}_{2}\left(\times 10^{-6}\right)$ : It influences the aluminum addition and, consequently, the aluminum-oxide content. It is measured with a temperature probe before the tapping.

- Lime $\mathrm{CaO}(\mathrm{kg})$ : It is added during the tapping and it helps create additional slag which is needed also during the ladle-furnace treatment.

- Calcium carbonate $(\mathrm{kg})$ : It is added during the tapping to reduce the slag density.

- Aluminum bricks $(\mathrm{kg})$ : They are added during the tapping in order to deoxidize the melt (the killed steel). Their amount depends on the active-oxygen content.
- The presence of the slag in the electric-arc furnace: The slag in the electric-arc furnace is the slag that was unintentionally poured into the ladle during the tapping. The electric-arc-furnace operator adjusts the tapping according to the melt quantity and tap-hole dimensions. The slag in the electric-arc furnace negatively influences the processing of the melt in the ladle furnace. Chemical and physical reactions evolve within a time shift or it can happen that they do not evolve at all.

- Aluminium wire $(\mathrm{m})$ : It is added to the ladle furnace according to the working instructions. It binds oxygen into aluminium oxides.

- CaSi wire (m): Calcium is added for melt modifying. It is proportional with the aluminium content. The modification is, in fact, the ability of calcium to bind unwanted inclusions and transport them into the slag.

$-t_{\mathrm{Al}-\mathrm{CaSi}}(\mathrm{min})$ : This is the time between the additions of aluminium and $\mathrm{CaSi}$ wires.

- Sulphur wire (m): It is added to form MnS inclusions which increase the machinability.

- The contents of $\mathrm{Al}, \mathrm{Ca}, \mathrm{S}$ at the beginning of the melt's ladle-furnace treatment $(\%)$

- The final contents of $\mathrm{Al}, \mathrm{Ca}$ and $\mathrm{S}(\%)$

$-\Delta T\left({ }^{\circ} \mathrm{C}\right)$ : This is the temperature difference between the actual and prescribed casting temperatures measured in the tundish.

$-n_{\mathrm{s}}$ : This is the number of the strands used during the casting. A continuous caster has three strands, but it can happen that the inner nozzle becomes clogged and the casting is, therefore, performed with fewer strands. Proportionally, the casting lasts longer and, consequently, the melt temperature drops influencing the casting speed.

- The casting speed $(\mathrm{m} / \mathrm{min})$ : It depends on the steel chemical composition and the melt temperature.

- The casting-speed variation $(\mathrm{m} / \mathrm{min})$ : Due to temperature drops the casting speed must be changed.

- $n_{\text {lno }}$ : This is the number of the ladle-nozzle openings with the oxygen lance.

- The rolled-bar diameter $(\mathrm{mm})$

Table 1: Experimental data

Tabela 1: Eksperimentalni podatki

\begin{tabular}{|c|c|c|c|c|c|c|c|c|c|c|c|c|c|c|c|c|c|c|c|c|c|c|}
\hline \multirow[b]{2}{*}{ 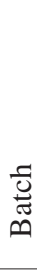 } & \multicolumn{4}{|c|}{$\begin{array}{c}\text { Electric-arc-furnace } \\
\text { parameters }\end{array}$} & \multicolumn{12}{|c|}{ Ladle-furnace parameters } & \multicolumn{5}{|c|}{ Casting parameters } & \multirow[b]{2}{*}{ 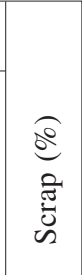 } \\
\hline & $\begin{array}{l}0 \\
0 \\
\times \\
0 \\
0\end{array}$ & 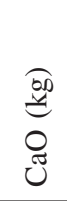 & 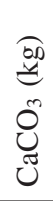 & 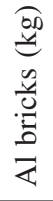 & $\frac{0_{0}^{ \pm}}{\infty}$ & 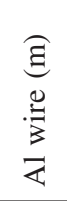 & 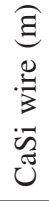 & 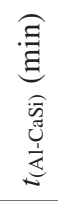 & $\begin{array}{l}\underset{\Xi}{\Xi} \\
\stackrel{0}{3} \\
\infty\end{array}$ & $\frac{\sqrt{2}}{\sqrt{2}}$ & $\underbrace{2}_{\bar{s}}$ & $\underbrace{\widehat{\theta}}_{\tilde{e}}$ & $\frac{2}{2}$ & $\underbrace{2}_{n}$ & 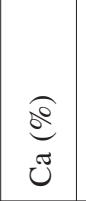 & $\underbrace{\underset{0}{0}}_{\forall}$ & 泀 & 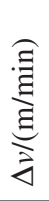 & 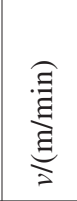 & 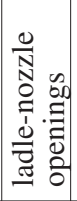 & 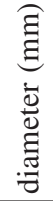 & \\
\hline 1 & 367.8 & 670 & 40 & 65 & 0 & 30 & 80 & & 110 & 0.013 & 0.02 & 0.005 & 0.028 & 0.027 & 0.025 & 40 & 3 & & & 0 & 37 & 27.42 \\
\hline 1 & 367.8 & 670 & 40 & 65 & 0 & 130 & 80 & & & 0.013 & 0.02 & 0.005 & 0.028 & 0.027 & 0.025 & 40 & 3 & 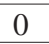 & & 0 & 44 & 33.29 \\
\hline 2 & 357.3 & 600 & 60 & 82 & 0 & 90 & 90 & 10 & & 0.01 & 0.045 & 0.003 & 0.029 & 0.029 & 0.035 & 55 & 3 & 0 & & 0 & 28 & 18.91 \\
\hline 2 & 357.3 & 600 & 60 & 82 & 0 & 90 & 90 & 10 & 105 & 0.01 & 0.045 & 0.003 & 0.029 & 0.029 & 0.035 & 55 & 3 & & $1.0 \mathrm{~J}$ & 0 & 33 & 30.92 \\
\hline 3 & 444.7 & 600 & 30 & 60 & 0 & 140 & 70 & 29 & 120 & 0.007 & 0.015 & 0.002 & 0.03 & 0.031 & 0.027 & 42 & 3 & 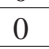 & 1.65 & 0 & 37 & 33.67 \\
\hline
\end{tabular}




\begin{tabular}{|c|c|c|c|c|c|c|c|c|c|c|c|c|c|c|c|c|}
\hline 4 & 38.5 & 600 & 0 & 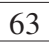 & & 65 & 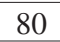 & 1 & \begin{tabular}{|l|l|l|l|l|l|l|}
140 & 0.025 & 0.034 & 0.002 & 0.025 & 0.034 & 0.026 \\
\end{tabular} & 39 & & 0 & 1.73 & & 28 & \\
\hline 4 & 38.5 & 600 & 0 & 53 & & 65 & 80 & 14 & \begin{tabular}{|l|l|l|l|l|l|l|l|l|l|l|l|l|l|l|}
140 & 0.025 & 0.034 & 0.002 & 0.025 & 0.034 & 0.026 \\
\end{tabular} & 39 & & 0 & .73 & & 30 & 19.22 \\
\hline 4 & 38.5 & 600 & 0 & 3 & & 65 & 80 & 14 & \begin{tabular}{|l|l|l|l|l|l|l|}
40 & 0.025 & 0.034 & 0.002 & 0.025 & 0.034 & 0.026 \\
\end{tabular} & 39 & & 0 & 73 & & 44 & 5.74 \\
\hline 5 & 620.7 & 600 & 0 & 0 & & 65 & 87 & 52 & \begin{tabular}{|l|l|l|l|l|l|l|}
130 & 0.021 & 0.026 & 0.002 & 0.027 & 0.03 & 0.022 \\
\end{tabular} & 44 & & 0 & 73 & & 28 & 12.88 \\
\hline 5 & 620.7 & 600 & 0 & 60 & & 65 & 37 & 52 & \begin{tabular}{|l|l|l|l|l|l|l|}
30 & 0.021 & 0.026 & 0.002 & 0.027 & 0.03 & 0.022 \\
\end{tabular} & 44 & & 0 & 73 & & 7 & 17.78 \\
\hline 6 & 210.1 & 600 & 0 & 60 & & 125 & 90 & 21 & \begin{tabular}{|l|l|l|l|l|l|l|}
35 & 0.019 & 0.021 & 0.004 & 0.023 & 0.024 & 0.03 \\
\end{tabular} & 45 & & 0 & 73 & & 30 & 15.69 \\
\hline 7 & 506.5 & 720 & 0 & 70 & & 70 & 96 & 17 & \begin{tabular}{|l|l|l|l|l|l|l|}
120 & 0.006 & 0.02 & 0.002 & 0.035 & 0.02 & 0.037 \\
\end{tabular} & 39 & & 0 & 73 & & 33 & 32.88 \\
\hline 8 & 203.3 & 600 & 0 & 50 & & 70 & 80 & 30 & \begin{tabular}{|l|l|l|l|l|l|l|}
60 & 0.021 & 0.045 & 0.001 & 0.034 & 0.029 & 0.028 \\
\end{tabular} & 46 & & 0.05 & .68 & & 44 & 6.58 \\
\hline 9 & 352.9 & 650 & 0 & 70 & & 70 & 90 & 45 & \begin{tabular}{|l|l|l|l|l|l|l|}
120 & 0.022 & 0.043 & 0.001 & 0.03 & 0.026 & 0.033 \\
\end{tabular} & 45 & & 0 & .73 & & 33 & 35.11 \\
\hline 9 & 352.9 & 650 & 0 & 70 & & 70 & 90 & 45 & \begin{tabular}{|l|l|l|l|l|l|l|}
120 & 0.022 & 0.043 & 0.001 & 0.03 & 0.026 & 0.033 \\
\end{tabular} & 45 & & 0 & 73 & & 37 & 32.16 \\
\hline 10 & 216.7 & 600 & 0 & 55 & & 20 & 85 & 10 & \begin{tabular}{|l|l|l|l|l|l|l|}
80 & 0.035 & 0.038 & 0.001 & 0.029 & 0.026 & 0.03 \\
\end{tabular} & 50 & & 0 & .68 & & 37 & 8.13 \\
\hline 11 & 767.2 & 600 & 40 & 70 & & 80 & 90 & 45 & 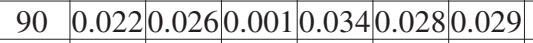 & 26 & & 0 & 83 & & 28 & 29.2 \\
\hline 11 & 767.2 & 600 & 0 & 70 & & 80 & 90 & 5 & \begin{tabular}{|l|l|l|l|l|l|l|l|l|l|l|l|l|l|}
90 & 0.022 & 0.026 & 0.001 & 0.034 & 0.028 & 0.029 \\
\end{tabular} & 26 & & 0 & 83 & & 33 & 31.88 \\
\hline 11 & 767.2 & 600 & 0 & 70 & & 80 & 90 & 5 & \begin{tabular}{|l|l|l|l|l|l|l|l|l|l|l|l|l|l|l|}
90 & 0.022 & 0.026 & 0.001 & 0.034 & 0.028 & 0.029 \\
\end{tabular} & 26 & & & 3 & & 37 & 23.81 \\
\hline 12 & 222.2 & 600 & 0 & 55 & & 90 & 90 & 0 & \begin{tabular}{l|l}
135 & 0.0 \\
\end{tabular} & 50 & & 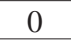 & 68 & & 30 & 58.5 \\
\hline 12 & 222.2 & 600 & 0 & 55 & & 90 & 90 & 30 & 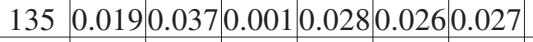 & 50 & & 0 & 68 & & 33 & 68.33 \\
\hline 12 & 222.2 & 600 & 0 & 55 & & 90 & 90 & or & \begin{tabular}{|l|l|l|l|l|l|l|l|l|l|l|l|}
135 & 0.019 & 0.037 & 0.001 & 0.028 & 0.026 & 0.027 \\
\end{tabular} & 50 & & 0 & .68 & & 44 & 26.85 \\
\hline 13 & 13.2 & 40 & 0 & 62 & & 0 & 90 & & \begin{tabular}{l|l}
70 & 0.0 \\
\end{tabular} & 45 & & 0.05 & 3 & & 37 & 6.87 \\
\hline 14 & 544.8 & 600 & & 7 & & 5 & 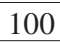 & 0 & \begin{tabular}{l|l}
140 & 0.0 \\
\end{tabular} & 40 & & 0 & 3 & & 14 & 37.67 \\
\hline 14 & 544.8 & 600 & 0 & 75 & & 105 & 10 & 40 & \begin{tabular}{|l|l|l|l|l|} 
& 0.029 & 0.025 & 0.033 \\
\end{tabular} & 40 & & 0 & & & 37 & 61.15 \\
\hline 15 & 513.6 & 600 & U & 45 & & 50 & 75 & 10 & \begin{tabular}{|l|l|l|l|l|l|l|}
130 & 0.021 & 0.03 & 0.003 & 0.03 & 0.024 & 0.031 \\
\end{tabular} & 53 & & 0 & 63 & & 44 & 33.39 \\
\hline 15 & 513.6 & 0 & & & & 50 & 7. & & \begin{tabular}{|l|l|l|l|l|l|l|}
130 & 0.021 & 0.03 & 0.003 & 0.03 & 0.024 & 0.031 \\
\end{tabular} & 53 & & & 3 & & 28 & 53.25 \\
\hline 16 & 560.1 & 50 & & . & & 75 & 8. & 1 & 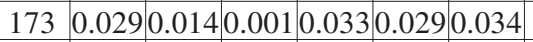 & 48 & & 05 & 8 & & 30 & 37.74 \\
\hline 16 & 560.1 & 760 & 0 & 60 & & 75 & 8. & 11 & $\begin{array}{lllll} & 0.033 & 0.029 & 0 .\end{array}$ & 48 & & 5 & $\delta$ & & 37 & 50.29 \\
\hline 16 & 560.1 & 760 & 0 & 60 & & 75 & 8. & 11 & 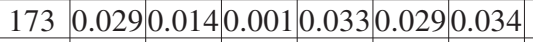 & 48 & & 0.05 & 8 & & 30 & 5.216 \\
\hline 17 & 163.6 & 00 & 0 & 5 & & 70 & 8. & & 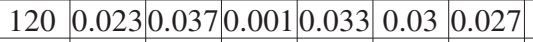 & 47 & & & 3 & & 14 & 23.76 \\
\hline 17 & 163.6 & 0 & & & & 70 & 8 & & \begin{tabular}{|l|l|l|l|l|l|l|}
120 & 0.023 & 0.037 & 0.001 & 0.033 & 0.03 & 0.027 \\
\end{tabular} & 47 & & & & & 37 & 63.09 \\
\hline 18 & 320.6 & 600 & & 62 & & 225 & 8 & 60 & \begin{tabular}{lll|l|l|l}
200 & 0.007 & 0.019 & 0.
\end{tabular} & 40 & & 0 & & & 28 & 4.732 \\
\hline 19 & 162.6 & 600 & 0 & 6 & & 0 & 80 & - & \begin{tabular}{|l|l|l|l|l|l|l|}
70 & 0.04 & 0.041 & 0.002 & 0.032 & 0.038 & 0.036 \\
\end{tabular} & 42 & & 0.12 & 5 & & 33 & 4.762 \\
\hline 20 & 317.8 & 600 & 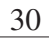 & 6 & & 90 & 80 & & 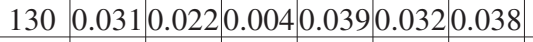 & 50 & & & 8 & & 8 & 11.27 \\
\hline 20 & 317.8 & 00 & & & & 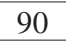 & 8 & & \begin{tabular}{l|l|l|l|}
30 & 0.031 & 0.0 \\
\end{tabular} & 50 & & & & & 30 & 6.41 \\
\hline 21 & 313 & 600 & & 6 & & 10 & 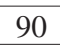 & & 350.0 & 46 & & & 3 & & 37 & 11.08 \\
\hline 21 & 313 & 600 & & 6 & & & 90 & & \begin{tabular}{l|l|l|l}
35 & 0.017 & 0.0 \\
\end{tabular} & 46 & & & & & 0 & 14.81 \\
\hline 21 & 313 & 600 & 40 & 6 & & & 96 & & \begin{tabular}{|l|l|l|l|}
2 & 0.028 & 0.023 & 0.031 \\
\end{tabular} & 46 & & & & & 7 & 27.12 \\
\hline 22 & 371.3 & 00 & & & & & 9 & & \begin{tabular}{|l|l|l|l|}
20 & 0.017 & 0.018 & 0.0 \\
\end{tabular} & 4( & & & & & 30 & 38.47 \\
\hline 22 & 371.3 & 0 & & 6 & & 115 & 93 & & 1200.0 & 40 & & & 3 & & 37 & 37.12 \\
\hline 23 & 327 & 600 & & 6 & & 0 & 9 & & \begin{tabular}{|l|l|l|l|l|l|l|}
105 & 0.032 & 0.02 & 0.003 & 0.028 & 0.027 & 0.041 \\
\end{tabular} & 40 & & & & & 3 & 68.78 \\
\hline 23 & 327 & 600 & 2 & 6 & & 2 & 9 & & \begin{tabular}{|l|l|l|l|l|l|l|}
105 & 0.032 & 0.02 & 0.003 & 0.028 & 0.027 & 0.041 \\
\end{tabular} & 4 & & & & & 7 & 46.86 \\
\hline 24 & 170.6 & 00 & & & & & 8 & & 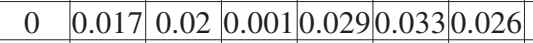 & 4 & & & & & 3 & 18.11 \\
\hline 25 & 630.6 & 0 & 0 & & & 12 & 0 & & \begin{tabular}{l|l|l|l}
33 & 0.012 & 0.0 \\
\end{tabular} & 4 & & & 3 & & 4 & 24.85 \\
\hline 25 & 630.6 & 600 & 3 & 7 & & & 8 & & \begin{tabular}{l|l|l|l|l|l}
133 & 0.012 & 0.036 & 0.005 & 0.
\end{tabular} & 48 & & & & & 37 & 25.43 \\
\hline 26 & 248 & 600 & 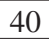 & & & & & & 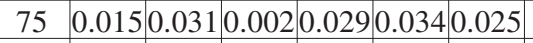 & 43 & & & & & 44 & 25.38 \\
\hline 27 & 355.7 & 0 & & 7 & & & 0 & & \begin{tabular}{|l|l|l|l|l|l|}
0.02 & 0.026 & 0.001 & 0.033 & 0.03 & 0.019 \\
\end{tabular} & 5 & & & & & 3 & 50 \\
\hline 27 & 355.7 & 0 & 3 & 7 & & & 8. & & \begin{tabular}{|l|l|l|l|l|l|}
0.02 & 0.026 & 0.001 & 0.033 & 0.03 & 0.019 \\
\end{tabular} & 51 & & & & & 7 & 64.21 \\
\hline 28 & 155.8 & 600 & 70 & 60 & & 1 & 8 & 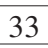 & \begin{tabular}{|l|l|l|l|l|l|l|}
120 & 0.01 & 0.03 & 0.001 & 0.029 & 0.035 & 0.024 \\
\end{tabular} & 42 & & 0 & & & 33 & 18.56 \\
\hline 28 & 155.8 & 600 & 7 & 6 & & & 8. & 33 & \begin{tabular}{|l|l|l|l|l|l|l|}
120 & 0.01 & 0.03 & 0.001 & 0.029 & 0.035 & 0.024 \\
\end{tabular} & 42 & & & & & 37 & 35.6 \\
\hline 29 & 205.6 & 600 & & 5 & & & , & 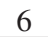 & \begin{tabular}{|l|l|l|l|l|l|l|}
107 & 0.011 & 0.04 & 0.002 & 0.033 & 0.023 & 0.03 \\
\end{tabular} & 4. & & & & & 4 & 22.81 \\
\hline 30 & 267.1 & 600 & 4 & 6 & & 50 & 7. & & \begin{tabular}{|l|l|l|l|l|l|l|l|}
95 & 0.025 & 0.033 & 0.001 & 0.03 & 0.027 & 0.031 \\
\end{tabular} & 4 & & 05 & 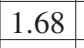 & & 37 & 23.84 \\
\hline 30 & 267.1 & 600 & 0 & 60 & 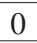 & 50 & 7. & 10 & \begin{tabular}{|l|l|l|l|l|l|l|}
95 & 0.025 & 0.033 & 0.001 & 0.03 & 0.027 & 0.031 \\
\end{tabular} & 46 & 3 & .05 & 68 & & 37 & 7.297 \\
\hline 31 & 348.4 & 600 & 4 & 60 & & 12 & 73 & 40 & \begin{tabular}{|l|l|l|l|l|l|l|l|l|l|l|l|} 
& 0.015 & 0.023 & 0.002 & 0.029 & 0.024 & 0.028 \\
\end{tabular} & 41 & & 0.05 & 68 & & 44 & 9.655 \\
\hline 32 & 652.6 & 650 & 4 & 8 & & 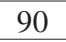 & 8 & & 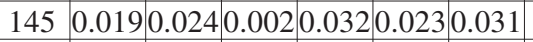 & 5 & & 0 & & & 30 & 8.411 \\
\hline 32 & 652.6 & 650 & 40 & 8 & & 90 & 8 & 1 & \begin{tabular}{|l|l|l|l|l|l|l|l|l|l|l|l|l|l|}
145 & 0.019 & 0.024 & 0.002 & 0.032 & 0.023 & 0.031 \\
\end{tabular} & 5 & & 0 & 8 & & 44 & 35.27 \\
\hline 33 & 184.8 & 600 & 30 & 55 & 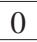 & 150 & 80 & 40 & \begin{tabular}{|l|l|l|l|l|l|l|l|l|l|l|l|l|l|l|l|l|l|}
115 & 0.01 & 0.022 & 0.002 & 0.031 & 0.029 & 0.032 \\
\end{tabular} & 45 & 3 & .05 & & & 30 & 16.29 \\
\hline 33 & 184.8 & 600 & 30 & 55 & 0 & & 8( & 4( & \begin{tabular}{|l|l|l|l|l|l|l|l|l|l|l|l|}
115 & 0.01 & 0.022 & 0.002 & 0.031 & 0.029 & 0.032 \\
\end{tabular} & 45 & & 0.05 & 68 & & 33 & 23.68 \\
\hline 34 & 455.4 & 600 & 30 & 58 & & & 9 & & 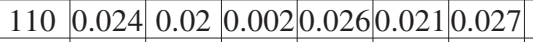 & 44 & & 0 & 3 & & 37 & 35.61 \\
\hline 34 & 455.4 & 600 & 30 & 58 & 0 & & 90 & $6 ?$ & 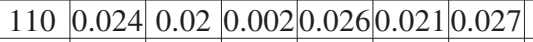 & 4 & & 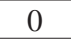 & & & 44 & 29.76 \\
\hline 34 & 455.4 & 600 & 30 & 58 & 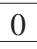 & 120 & 90 & 05 & \begin{tabular}{|l|l|l|l|l|l|l|l|l|l|l|l|l|l|l|l|l|}
110 & 0.024 & 0.02 & 0.002 & 0.026 & 0.021 & 0.027 \\
\end{tabular} & 44 & & & 1.73 & & 37 & 35.03 \\
\hline 34 & 455.4 & 600 & 30 & 58 & 0 & 120 & 90 & 63 & \begin{tabular}{|l|l|l|l|l|l|l|}
110 & 0.024 & 0.02 & 0.002 & 0.026 & 0.021 & 0.027 \\
\end{tabular} & 44 & 3 & 0 & 1.73 & 1 & 44 & 38.83 \\
\hline
\end{tabular}




\section{MODELING OF OCCURRENCE OF SURFACE DEFECTS WITH GENETIC PROGRAMMING}

Genetic programming is probably the most general evolutionary optimization method. ${ }^{15-19}$ The organisms that undergo an adaptation are in fact mathematical expressions (models) for a nozzle-opening prediction consisting of the available function genes (i.e., the basic arithmetical functions) and terminal genes (i.e., independent input parameters and random floating-point constants). In our case the models consist of the function genes of addition $(+)$, subtraction $(-)$, multiplication $(\times)$ and division $(/)$ and terminal genes of active oxygen $\mathrm{O}_{2}$ $\left(\mathrm{O}_{2}\right)$, lime $(\mathrm{CaO})$, calcium carbonate $\left(\mathrm{CaCO}_{3}\right)$, aluminum bricks $(A l b)$, the electric-arc-furnace slag presence (slag), the aluminium wire (Alw), the CaSi wire (CaSiw), the time between the additions of aluminium and $\mathrm{CaSi}$ wires $(t \mathrm{Al}-\mathrm{CaSi})$, the sulphur wire $(\mathrm{Sw})$, the contents of Al $(\mathrm{All}), \mathrm{Ca}(\mathrm{Cal})$ and $\mathrm{S}(\mathrm{Sl})$ at the beginning of the melt's ladle-furnace treatment, the final contents of $\mathrm{Al}$ $(\mathrm{Al}), \mathrm{Ca}(\mathrm{Ca})$ and $\mathrm{S}(\mathrm{S})$, the temperature difference between the actual and prescribed casting temperatures measured in the tundish $(\mathrm{d} T)$, the number of strands used during the casting $(n s)$, the casting speed $(v c)$, the casting-speed variation $(v v c)$, the number of ladle-nozzle openings with the oxygen lance (nlno) and the rolled-bar diameter $(d)$.

Random computer programs of various forms and lengths are generated by means of selected genes at the beginning of the simulated evolution. Afterwards, the varying of the computer programs during several iterations, known as generations, is performed by means of genetic operations. For the progress of the population only the reproduction and crossover are sufficient. After the completion of the variation in the computer programs a new generation is obtained that is evaluated and compared with the experimental data.

The process of changing and evaluating the organisms is repeated until the termination criterion of the process is fulfilled. This is the prescribed maximum number of generations.

For the process of simulated evolutions the following evolutionary parameters were selected: the population of organisms: 500, the greatest number of generations: 100, the reproduction probability of 0.4 , the crossover probability of 0.6 , the greatest permissible depth when creating a population: 6 , the greatest permissible depth after the operation of a crossover of two organisms: 10, and the smallest permissible depth of organisms when generating new organisms: 2. Genetic operations of the reproduction and crossover were used. For the selection of organisms the tournament method with a tournament size of 7 was used. For the model fitness, the average square of deviation from the monitored data was selected. It is defined as:

$$
\Delta=\frac{\sum_{i=1}^{n} \Delta_{i}^{2}}{n}
$$

where $n$ is the size of the monitored data and $\Delta_{i}$ is the square of deviation of a single sample data. The deviation of a single sample data, produced by an individual organism, is simply:

$$
\Delta_{i}=E_{i}-G_{i}
$$

where $E_{i}$ and $G_{i}$ are the actual and the predicted scrap fractions (which depend only on surface defects), respectively.

We developed 100 independent civilizations of mathematical models for the scrap-fraction prediction. Each civilization has the most succesfull organism - the mathematical model for the scrap-fraction prediction. The best most succesfull organism from all of the civilizations is presented here:

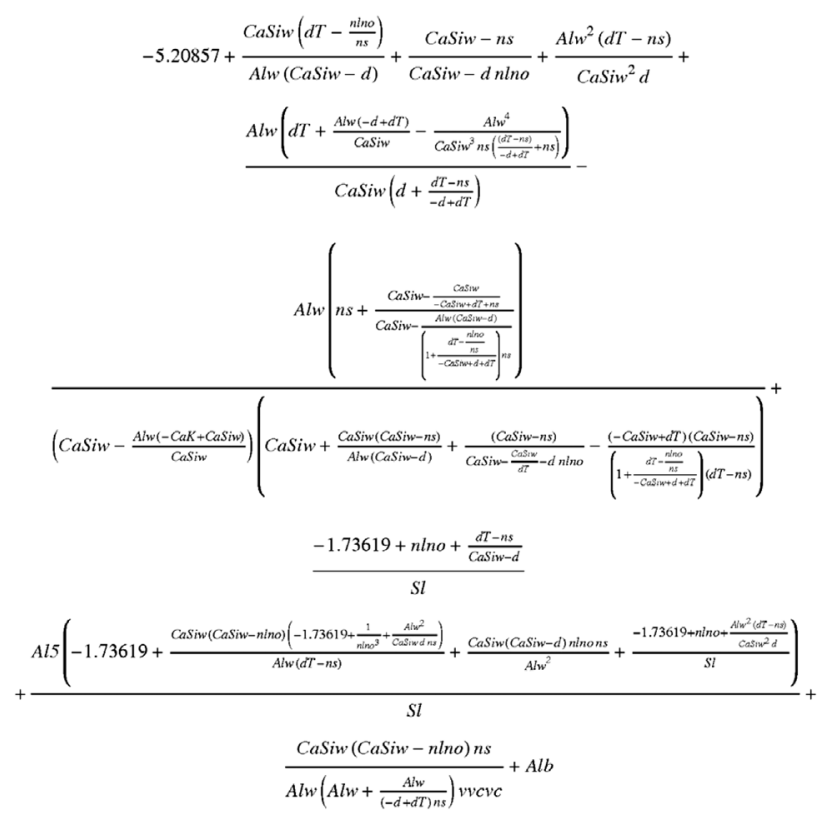

with the average relative deviation of $12.03 \%$.

The randomly driven process builds the fittest and most complex models from generation to generation and uses the ingredients that are most suitable for the experimental environment adaptation. For curiosity's sake, an analysis of the genes (parameters) excluded from the models is presented in Figure 2.

On the basis of the number of genes excluded from 100 obtained mathematical models we can assume the influence of the parameters on the scrap fraction. It is clear from the figure that out of 100 genetically obtained mathematical models only 12 models do not include the parameter of aluminium blocks and only 3 out of 100 models do not include the parameter of the number of ladle-nozzle openings. So, we can deduct that aluminium blocks and ladle-nozzle openings with an oxygen lance 


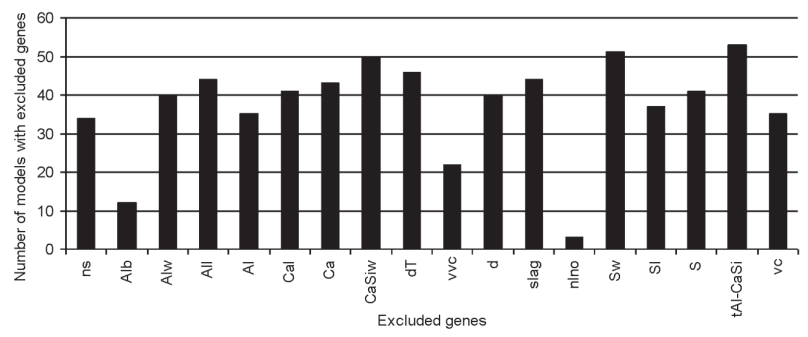

Figure 2: Frequency of the genes excluded from the best 100 mathematical models for scrap fraction

Slika 2: Frekvenca izločenih genov na podlagi najboljših 100 matematičnih modelov za delež izmeta

are probably the most important parameters influencing the occurrence of surface defects.

\section{PRACTICAL IMPLEMENTATION OF THE MODELING RESULTS}

At the beginning of 2011, the aluminium-calciumfree (ACF) steelmaking technology was used for the C45 steel instead of the aluminium-killed steelmaking technology. The data for the analysis was collected on the basis of 17 consecutively inspected batches of the C45 steel (automatic control line) in Štore Steel Ltd. (Table 2).

Table 2: Automatic-control-line results (scrap fractions) for 17 consecutively inspected batches (automatic control line) of C45 steel made with aluminium-calcium-free steelmaking technology

Tabela 2: Rezultati samodejne kontrolne linije (delež izmeta) za 17 zaporedno pregledanih šarž jekla C45, izdelanih po tehnologiji aluminium-calcium-free

\begin{tabular}{|c|c|}
\hline Batch & Scrap \% \\
\hline 1 & 0 \\
\hline 2 & 1.036 \\
\hline 2 & 2.102 \\
\hline 3 & 2.042 \\
\hline 3 & 2.809 \\
\hline 4 & 0.642 \\
\hline 5 & 0.877 \\
\hline 6 & 2.889 \\
\hline 7 & 0.902 \\
\hline 8 & 1.731 \\
\hline 9 & 1.512 \\
\hline 10 & 3.218 \\
\hline 11 & 1.570 \\
\hline 12 & 2.345 \\
\hline 13 & 0.520 \\
\hline 14 & 0.700 \\
\hline 15 & 4.757 \\
\hline 16 & 1.086 \\
\hline 17 & 5.695 \\
\hline
\end{tabular}

The data was analyzed using Microcoft Excel. The $\alpha$ parameter was set at $\alpha=0.05$.

The t-test for unequal variances was used to compare the differences between the two populations. The results of the comparison of the automatic control results ob- tained during the aluminium-killed steelmaking period (Table 1) and aluminium-calcium-free steelmaking period (Table 2) are presented in Table 3.

Table 3: Comparison of automatic-control results obtained during aluminium-killed steelmaking period (Table 1) and aluminium-calciumfree steelmaking period (Table 2)

Tabela 3: Primerjava rezultatov samodejne kontrolne linije, pridobljenih v obdobju izdelave jekla na podlagi tehnologije z dodajanjem aluminija (tabela 1) in med obdobjem uporabe tehnologije aluminium-calcium-free (tabela 2 )

\begin{tabular}{|c|c|c|}
\hline & $\begin{array}{c}\text { Al-killed } \\
\text { steelmaking }\end{array}$ & ACF steelmaking \\
\hline Mean & 68.45302471 & 1.917526316 \\
\hline Variance & 260.6865252 & 2.146430596 \\
\hline $\begin{array}{c}\text { Observations } \\
\begin{array}{c}\text { Hypothesized mean } \\
\text { difference }\end{array}\end{array}$ & 61 & 19 \\
\hline $\mathrm{d} f$ & 63 & \\
\hline $\mathrm{t}-$ stat & 31.76825617 & \\
\hline$P(T<=t)$ one-tail & $8.65978 \mathrm{E}-41$ & \\
\hline$t$ critical one-tail & 1.669402222 & \\
\hline$P(T<=t)$ two-tail & $1.73196 \mathrm{E}-40$ & \\
\hline$t$ critical two-tail & 1.998340522 & \\
\hline
\end{tabular}

t-test: two-sample assuming unequal variances

The comparison shows that there is a statistically significant difference between the batches made within the aluminium-killed steelmaking period and aluminium-calcium-free steelmaking period $(p<0.05)$. The batches from the aluminium-calcium-free steelmaking period statistically have a significantly smaller amount of surface defects (scrap percenatage).

\section{CONCLUSION}

The purpose of this paper was to reduce surface defects (scrap fraction) on the $\mathrm{C} 45$ steel. The influences of the casting-temperature changes, the total oxygen, the number of added aluminum rods, the chemical analyses before and after steelmaking, added lime, aluminumcored wire, calcium-silicon-cored wire, sulphur-cored wire, the rolling dimensions, the casting speed, the opening of the ladle nozzle with oxygen on the surface defects of rolled bars were analyzed. The data for the analysis was collected on the basis of 34 consecutively inspected (automatic control line) batches of the C45 steel in Šrore Steel Ltd. The data was taken from the technological documentation of the cast batches and from the chemical archive. Afterwards a model for a scrap-fraction prediction was developed with the genetic-programming method allowing an evolution of better and better model variants during the simulation. There were 100 different models and only the best was used for the scrap-fraction prediction. The relative average deviation of the actual scrap fraction from the predicted scrap fraction was $12.03 \%$. Also, the frequencies of the genes excluded from the best 100 mathematical 
models for the scrap fraction were analyzed. The results show that the most influential parameters for the surface defects occurring on the $\mathrm{C} 45$ steel are the opening of the ladle nozzle with the oxygen lance and aluminum (blocks). According to the results, the steelmaking technology was changed. At the end of 2009 and at the beginning of 2010 the aluminium-calcium-free (ACF) steelmaking technology was used with the C45 steel instead of the aluminium-killed steelmaking technology. The t-test for unequal variances was used to compare the differences between the automatic-control results obtained during the aluminium-killed steelmaking period and the aluminium-calcium-free steelmaking period. The batches from the aluminium-calcium-free steelmaking period statistically had a significantly smaller amount of surface defects (scrap percenatage). The scrap fraction was reduced from the average of $68.45 \%$ to $1.92 \%$ - by more than 35 times. In future the implemented procedure can be applied to several steel grades.

\section{REFERENCES}

${ }^{1}$ W. R. Irving, Continuous casting of steel, Institute of Materials, London 1993

${ }^{2}$ C. Reilly, N. R. Green, M. R. Jolly, The present state of modeling entrainment defects in the shape casting process, Applied Mathematical Modelling, 37 (2013) 3, 611-628, doi:10.1016/j.apm.2012.04. 032

${ }^{3}$ J. Stetina, T. Mauder, L. F. Klimes, Increasing the surface temperature during the straightening of a continuously cast slab, Mater. Tehnol., 47 (2013) 3, 311-316

${ }^{4}$ Q. Lu, R. Yang, X. Wang, J. Zhang, W. Wang, Water modeling of mold powder entrapment in slab continuous casting mold, Journal of University of Science and Technology Beijing, Mineral, Metallurgy, Material, 14 (2007) 5, 399-404, doi:10.1016/S1005-8850(07) 60079-6

${ }^{5}$ N. Cheung, A. Garcia, The use of a heuristic search technique for the optimization of quality of steel billets produced by continuous casting, Engineering Applications of Artificial Intelligence, 14 (2001) 2, 229-238, doi:10.1016/S0952-1976(00)00075-0

${ }^{6}$ N. Cheung, C. A. Santos, J. A. Spim, A. Garcia, Application of a heuristic search technique for the improvement of spray zones cooling conditions in continuously cast steel billets, Applied Mathematical Modelling, 30 (2006) 1, 104-115, doi:10.1016/j.apm.2005.03. 008
${ }^{7}$ G. O. Tirian, I. Filip, G. Proştean, Adaptive control system for continuous steel casting based on neural networks and fuzzy logic, Neurocomputing, 125 (2014), 236-245, doi:10.1016/j.neucom.2012. 11.052

${ }^{8}$ M. S. Kulkarni, A. Subash Babu, Managing quality in continuous casting process using product quality model and simulated annealing, Journal of Materials Processing Technology, 166 (2005) 2, 294-306, doi:10.1016/j.jmatprotec.2004.09.073

${ }^{9}$ A. Sanz-García, A. V. Pernía-Espinoza, R. Fernández-Martínez, F. J. Martínez-de-Pisón-Ascacíbar, Combining genetic algorithms and the finite element method to improve steel industrial processes, Journal of Applied Logic, 10 (1012) 4, 298-308, doi:10.1016/j.jal.2012.07. 006

${ }^{10}$ M. S. Kulkarni, A. S. Babu, Optimization of Continuous Casting Using Simulation, Materials and Manufacturing Processes, 20 (2005) 4, 595-606, doi:10.1081/AMP-200041874

${ }^{11}$ M. Hrelja, S. Klancnik, T. Irgolic, M. Paulic, Z. Jurkovic, J. Balic, M. Brezocnik, Particle swarm optimization approach for modelling a turning process, Advances in Production Engineering \& Management, 9 (2014) 1, 21-30, doi:10.14743/apem2014.1.173

${ }^{12}$ M. Hrelja, S. Klancnik, J. Balic, M. Brezocnik, Modelling of a turning process using the gravitational search algorithm, International Journal of Simulation Modelling, 13 (2014) 1, 30-41, doi:10.2507/ IJSIMM13(1)3.248

${ }^{13}$ M. Chandrasekaran, D. Devarasiddappa, Artificial neural network modeling for surface roughness prediction in cylindrical grinding of Al-SiCp metal matrix composites and ANOVA analysis, Advances in Production Engineering \& Management, 9 (2014) 2, 59-70, doi:10.14743/apem2014.2.176

${ }^{14}$ X. W. Huang, X. Y. Zhao, X. L. Ma, An improved genetic algorithm for job-shop scheduling problem with process sequence flexibility, International Journal of Simulation Modelling, 13 (2014) 4, 510-522, doi:10.2507/IJSIMM13(4)CO20

${ }^{15}$ M. Kovačič, B. Jurjovec, L. Krajnc, Ladle nozzle opening and genetic programming, Mater. Tehnol., 48 (2014) 1, 23-26

${ }^{16} \mathrm{M}$. Kovačič, B. Šarler, Application of the genetic programming for increasing the soft annealing productivity in steel industry, Materials and Manufacturing Processes, 24 (2009) 3, 369-374, doi:10.1080/ 10426910802679634

${ }^{17}$ M. Kovačič, S. Senčič, Modeling of PM10 emission with genetic programming, Mater. Tehnol., 46 (2012) 5, 453-457

${ }^{18}$ M. Kovačič, P. Uratnik, M. Brezočnik, R. Turk, Prediction of the bending capability of rolled metal sheet by genetic programming, Materials and Manufacturing Processes, 22 (2007) 5, 634-640, doi:10.1080/10426910701323326

${ }^{19}$ J. R. Koza, Genetic programming III, Morgan Kaufmann, San Francisco 1999 